\title{
Tissue iron quantification in chronic liver diseases using MRI shows a relationship between iron accumulation in liver, spleen, and bone marrow
}

\author{
M. França a,b,*, L. Martí-Bonmatî́ ${ }^{\mathrm{c}}$, G. Porto ${ }^{\mathrm{b}, \mathrm{d}, \mathrm{e}}$, S. Silva ${ }^{\mathrm{f}}$, S. Guimarães ${ }^{\mathrm{g}}$, \\ Á. Alberich-Bayarri ${ }^{\mathrm{C}}$, J.R. Vizcaíno ${ }^{\mathrm{h}}$, H. Pessegueiro Miranda ${ }^{\mathrm{e}, \mathrm{i}, \mathrm{j}}$ \\ a Imaging Department - Centro Hospitalar do Porto, Largo Prof Abel Salazar, 4099-001 Porto, Portugal \\ b i3S, Instituto de Investigação e Inovação em Saúde, IBMC, Institute for Molecular and Cell Biology, Rua Alfredo Allen, \\ 208, 4200-135 Porto, Portugal \\ ${ }^{\mathrm{c}}$ Radiology Department, Hospital Universitario y Politécnico La Fe and Biomedical Imaging Research Group \\ (GIBI230), Av. Fernando Abril Martorell 106 Torre E, 46026 Valencia, Spain \\ ${ }^{\mathrm{d}}$ Haematology Department, Centro Hospitalar do Porto, Largo Prof Abel Salazar, 4099-001 Porto, Portugal \\ e Instituto de Ciências Biomédicas de Abel Salazar (ICBAS), University of Porto, Rua de Jorge Viterbo Ferreira, 228, \\ 4050-313 Porto, Portugal \\ ${ }^{\mathrm{f}}$ Faculty of Sciences, University of Porto, Rua do Campo Alegre, 4169-007 Porto, Portugal \\ g Pathology Department, Centro Hospitalar de S. João, Alameda Prof. Hernâni Monteiro, 4200-319 Porto, Portugal \\ h Pathology Department - Centro Hospitalar do Porto, Largo Prof Abel Salazar, 4099-001 Porto, Portugal \\ i Liver and Pancreas Transplantation Unit and Medicine Department - Centro Hospitalar do Porto, Largo Prof Abel \\ Salazar, 4099-001 Porto, Portugal \\ ${ }^{\mathrm{j}}$ Epidemiology Research Unit (EPIUnit), Institute of Public Health of the University of Porto, Rua das Taipas, 135, \\ 4050-600 Porto, Portugal
}

\section{ARTICLE INFORMATION}

\section{Article history:}

Received 20 February 2017

Received in revised form

10 July 2017

Accepted 31 July 2017
AIM: To investigate iron loading within the liver, pancreas, spleen, and bone marrow using magnetic resonance imaging (MRI) transverse relaxation rate $\left(R 2^{*}\right)$, in patients with diffuse liver diseases; to evaluate the relationships between iron accumulation in these tissue compartments; and to assess the association between tissue iron overload and the pattern of hepatic cellular iron distribution (hepatocytes versus Kupffer cells).

MATERIAL AND METHODS: Fifty-six patients with diffuse liver diseases had MRI-derived R2* values, using a multi-echo chemical-shift encoded MRI sequence, of the liver, pancreas, spleen, and vertebral bone marrow. All patients had liver biopsy samples scored for hepatic iron grading (0-4) and iron cellular distribution (within hepatocytes only or within both hepatocytes and Kupffer cells).

RESULTS: Liver R2* increased with histological iron grade $\left(\mathrm{R}_{S}=0.58, p<0.001\right)$ and correlated with spleen $\left(R_{S}=0.71, p<0.001\right)$ and bone marrow $R 2^{*}\left(R_{S}=0.66, p<0.001\right)$, but not with pancreatic $\mathrm{R}^{*}\left(\mathrm{R}_{\mathrm{S}}=0.22, p=0.096\right)$. Splenic and bone marrow $\mathrm{R}^{*}$ values were also correlated $\left(R_{S}=0.72, p<0.001\right)$. Patients with iron inside Kupffer cells had the highest $R 2^{*}$ in liver, spleen and bone marrow.

\footnotetext{
* Guarantor and correspondent: M. França, Imaging Department - Centro Hospitalar do Porto, Largo Prof Abel Salazar, 4099-001 Porto, Portugal. Tel.: +351222077500.

E-mail address: mariamanuela.franca@gmail.com (M. França).
} 
CONCLUSIONS: Patients with chronic diffuse liver diseases have concomitant hepatic, splenic, and bone marrow iron loading. The highest hepatic iron scores and iron inside Kupfer cells were associated with the highest splenic and bone marrow deposits, suggesting systemic iron accumulation in the mononuclear phagocytic system.

(c) 2017 The Royal College of Radiologists. Published by Elsevier Ltd. All rights reserved.

\section{Introduction}

Excessive accumulation of iron in the liver is mainly found in genetic haemochromatosis, transfusion-related haemosiderosis, and other iron-loading anaemias as a consequence of systemic iron overload. Hepatic iron overload also occurs in chronic hepatopathies due to local iron dyshomeostasis, its presence being associated to progression and worse prognosis of the underlying liver disease. ${ }^{1-3}$ Histopathologically, iron can be present within the hepatocytes and/or inside the Kupffer cells of the mononuclear phagocytic system (MPS). Within the hepatocytes, iron excess leads to oxidative stress and hepatocellular necrosis, a condition known as sideronecrosis. ${ }^{3}$ In these patients, iron accumulation in the MPS is associated with a more severe liver disease. ${ }^{1,4,5}$

Iron overload is recognised on magnetic resonance imaging (MRI) due to its lowering of the tissue signal intensity. Iron deposits within the liver and pancreas are characteristic of severe hereditary haemochromatosis; a pattern of liver, spleen, and bone marrow overload relates to iron inside the MPS cells; while isolated liver deposition is classically associated to milder forms of hereditary haemochromatosis or to chronic liver diseases. ${ }^{6,7}$ However, patients with chronic diffuse liver diseases and no history of blood transfusions often show a wide spectrum of iron distribution within liver, pancreas, spleen, and bone marrow in MRI studies, suggesting that iron accumulation may not be limited only to the liver.

Quantitative MRI assessment of liver iron overload by means of transverse relaxation rates (R2*) relaxometry has emerged as a safe, non-invasive, and accurate alternative to liver biopsy for the evaluation and follow-up of patients with hereditary haemochromatosis and transfusion-related haemosiderosis. ${ }^{2,8} \mathrm{R} 2^{*}$ relaxometry uses a series of images acquired with a multi-echo gradient echo sequence, with increasing echo time (TE). This sequence can be performed within a single breath-hold acquisition. The liver signal intensity is modelled as a multi-exponential periodic complex function of TE and the signal decay rate is then calculated. ${ }^{2}$ The greater the liver iron concentration, the higher the relaxation rate $\left(\mathrm{R} 2^{*}\right)^{2,8}$ In comparison to other MRI methods, such as R2 relaxometry or signal intensity ratio methods, $\mathrm{R} 2 *$ relaxometry performed with multi-echo chemical shift-encoded sequences has the advantage of a faster acquisition with simultaneous quantification of hepatic iron and fat.
Furthermore, MRI-derived R2* calculations have also been used to assess iron in other organs, such as the pancreas, spleen, and vertebral bone marrow, in patients with thalassemia, other iron-loading anaemias and primary haemochromatosis, providing new insights into the pathophysiology of systemic iron distribution ${ }^{9,10}$; however, to the authors' knowledge, a combined evaluation of the ironrelated R2* changes in the liver, pancreas, spleen, and bone marrow has never been established in the context of chronic diffuse liver diseases.

The aims of the present study were to quantitatively assess liver, pancreas, spleen, and bone marrow iron deposits and distribution, by means of the $\mathrm{R}^{*}$ values, in a series of patients with chronic diffuse liver diseases and liver biopsy. A secondary aim was to evaluate the relationship among R2* measurements in the different organs and to assess the relationship between tissue iron overload and the intracellular hepatic iron distribution (hepatocytes and/ or Kupffer cells).

\section{Materials and methods}

The Institutional Review Board approved this prospective single centre study. All recruited patients gave their written informed consent to participate. Over a period of 1 year, all consecutive patients with diffuse liver disease who underwent clinically indicated liver biopsy, were scheduled for a liver MRI examination. For the purpose of this study, patients with a diagnosis of hereditary haemochromatosis $(n=8)$ and liver transplant $(n=32)$ were excluded from further analyses. In addition, patients with severe pancreatic atrophy and diffuse pancreatic duct dilation $(n=2)$ or with movement-related MRI artefacts $(n=1)$ were also excluded. No patient had a history of chronic blood transfusion. The final study population included 56 patients (30 men and 26 women), with a mean age of 44.7 (standard deviation, 13.8; range, 19-77) years.

The histological and laboratory-based aetiology of the liver diseases was chronic hepatitis $C$ virus infection $(n=15)$, alcoholic liver disease $(n=6)$, autoimmune chronic hepatitis $(n=15)$, chronic hepatitis B virus infection $(n=5)$, nonalcoholic fatty liver disease $(n=9)$, and non-specific chronic hepatitis $(n=6)$. Serum ferritin values were retrieved from the patient's clinical data files. Because ferritin values may change over time, only ferritin measurements from within 30 days from liver biopsy and MRI examination were considered for this study ( $n=37$ patients). 


\section{Liver biopsy and histopathological analysis}

Percutaneous liver biopsy samples were acquired under ultrasonography guidance (16-18 G needles). Biopsy was used as the reference standard for grading hepatic iron deposits. A pathologist (S.G., more than 10 years' experience), blinded to the MRI results, evaluated the biopsy samples using haematoxylin and eosin and Perls' Prussian stains for iron grading. ${ }^{11}$ Furthermore, the cellular stainable iron distribution compartment was classified as within hepatocytes only or within both hepatocytes and Kupffer cells.

MRI

Most of the patients (53 patients, 95\%) underwent MRI examination the same day as liver biopsy, and all patients had a liver MRI examination performed within 21 days from the liver biopsy (mean time 2.2 days, interquartile range 1 day). The MRI studies were performed at a single institution with 3 T MRI equipment (TX Achieva, Philips Healthcare, the Netherlands), using a 16-channel phased-array surface coil. A two-dimensional (2D) multi-echo chemical shift encoded sequence was performed (12 echoes, echo times $[\mathrm{TEs}]=0.99$ to 8.69 , short echo spacing $=0.7 \mathrm{~ms}$; repetition time $[\mathrm{TR}]=10 \mathrm{~ms} ; 10^{\circ}$ flip angle; 34 section; $3 \times 3 \mathrm{~mm}$ voxel dimensions; $7 \mathrm{~mm}$ section thickness; $0.3 \mathrm{~mm}$ gap; $2 \times 2 \mathrm{~mm}$ reconstruction voxel size; $375 \times 302 \mathrm{~mm}$ field of view; 1.8 parallel imaging effective acceleration factor; $2433 \mathrm{~Hz} /$ pixel bandwidth). The total acquisition time for imaging the upper abdomen under end-expiratory phase single breathhold acquisition never exceeded 15 seconds.

Quantification of iron-related R2* measurements was performed using QLiver software (QUIBIM, Valencia, Spain; Fig 1), which is based on least squares analysis by Levenberg-Marcquardt algorithm, being used for the simultaneous quantification of $\mathrm{R}^{*}$ and proton density fat fraction. ${ }^{12}$ MRI images were evaluated by a trained research student (S.S., 3 months of experience performing liver MRI measurements) under the supervision of an experienced abdominal radiologist (M.F., 10 years of experience). Both readers were blinded to histopathological results. Liver iron-related $\mathrm{R} 2 *$ values $\left(\mathrm{s}^{-1}\right)$ were calculated as the average measurements of eight circular regions of interest (ROIs) of $8 \mathrm{~mm}$ diameter. These ROIs were manually placed in the right hepatic segments, avoiding vessels and lesions. Pancreatic R2* values were estimated as the average of $6 \mathrm{~mm}$ diameter ROIs, placed within the parenchyma at the head, body, and tail of the pancreas. Splenic R2* values were calculated as the average of three measurements obtained with $8 \mathrm{~mm}$ circular ROIs located in the splenic parenchyma. Vertebral bone marrow R2* values were estimated as the average values of three $8 \mathrm{~mm}$ ROIs, manually placed in the body of the $12^{\text {th }}$ dorsal to the second lumbar vertebras. All $\mathrm{R}^{*}$ measurements were done in $<5$ minutes per patient. The interobserver and the inter-examination agreement for the different $\mathrm{R} 2^{*}$ measurements were previously assessed in 21 healthy volunteers (12 males, nine females; age mean \pm SD, $38.1 \pm 16$ years), who were scanned on two

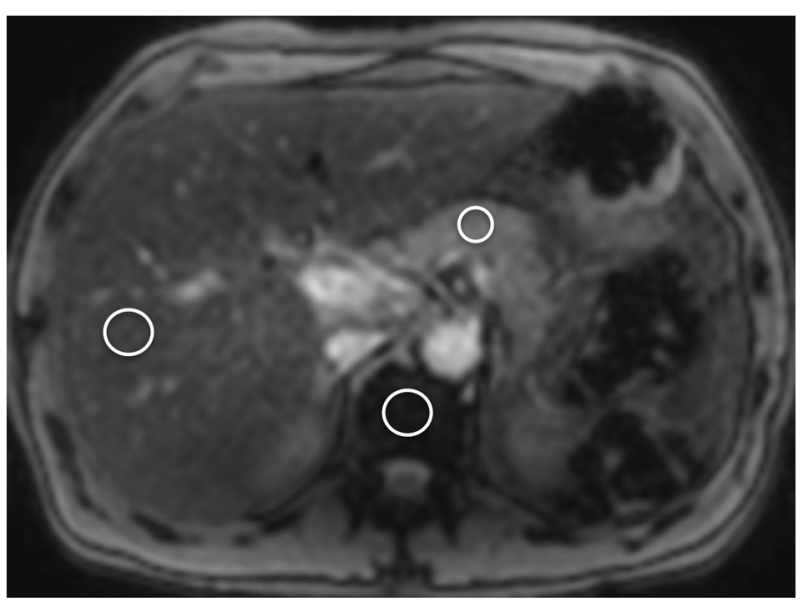

(a)

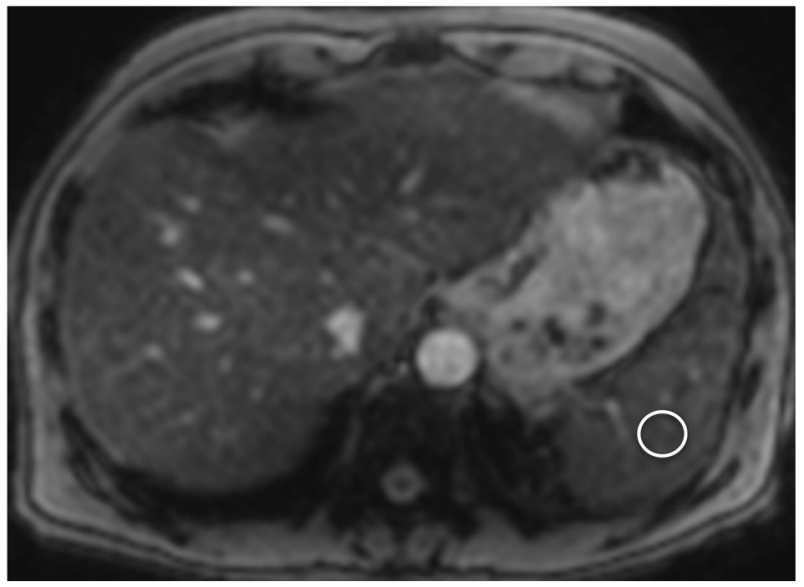

(b)

Figure $1 \mathrm{R} 2 *$ values estimated in a 56-year-old male patient with chronic HCV infection and hyperferritinaemia (1902 ng/ml). Liver biopsy revealed histological iron grade 2. (a-b) Axial abdominal MRI images show ROIs in the liver, spleen, vertebra bone marrow, and pancreas, estimating $R 2^{*}$ values of $101,108,265$, and $23 \mathrm{~s}^{-1}$, respectively.

different sessions at the same day and images evaluated by two readers. R2* measurements in the liver, spleen, and bone marrow showed strong agreement between the two observers and between the two different MRI examinations (intra-class correlation coefficients higher than 0.95). In addition, pancreatic $\mathrm{R}^{*}$ measurements showed good agreement between observers and different examinations (intra-class correlation coefficients higher than 0.78).

\section{Statistical analysis}

Descriptive statistics were used to summarise the patients' characteristics. Categorical variables were evaluated by Fisher's exact test. Group data are presented as mean and standard deviation, or median and interquartile range. All the R2* values distributions were non-symmetrical, even after several transformations. Differences of R2* values between histological groups of hepatic iron loading and between groups of different cellular iron distribution 
compartments (iron only in hepatocytes versus iron present in Kupffer cells) were assessed using Kruskal-Wallis test with post-hoc Mann-Whitney test and Bonferroni correction. The reported $p$-value, which is multiplied by the number of comparisons (three comparisons), reflects the Bonferroni adjustment.

Spearman's correlation coefficient was used to correlate liver, pancreas, spleen, and vertebral bone marrow R2*, and also ferritin values. Spearman's correlation coefficient was also used to calculate the degree of association between continuous (liver, pancreas, spleen, and vertebral bone marrow R2* measurements, and ferritin values) and ordinal variables (liver histological iron scores). SPSS (version 24; SPSS, IBM, Chicago, IL, USA) was used for the analysis.

A $p<0.05$ was considered as statistically significant for all tests, including the Bonferroni adjusted $p$-values in multiple comparisons.

\section{Results}

\section{Histopathological and laboratory results}

In the 56 liver specimens, 25 (45\%) patients had no iron deposits identified at histological examination (grade 0 ). On the contrary, 31 (55\%) had hepatic iron overload. Of these, 19 (61\%) patients were classified as grade 1, 10 (32\%) patients were grade 2 , and two (7\%) patients were grade 3 . For statistical analysis, iron histological grades were grouped as grades $2-3$ (12 patients, 39\%).

The main cellular distribution of iron deposits was registered in 21 of the 31 patients with iron overload: nine (43\%) samples had iron only within hepatocytes and 12 (57\%) cases had iron within both hepatocytes and Kupffer cells. This classification could not be established in 10 patients due to pathological sample degradation, and these were considered "missing values". There was an association between the histological hepatic iron score and the histological cellular pattern of iron distribution. Stainable iron within the Kupffer cells was more frequent in patients with higher liver iron scores whereas only one patient with high iron overload had iron only within hepatocytes (Fischer's exact test, $p=0.01$; Spearman's rank correlation coefficient, $\mathrm{R}_{\mathrm{S}}=0.63, p=0.002$; Table 1 ). There was no significant association between the aetiology of liver diseases and the main histological cellular pattern of iron distribution (Fischer's exact test, $p=0.29$ ).

Table 1

Distribution of patients according to the histological hepatic iron score and the histological cellular pattern of iron distribution (available in 21 of the 31 patients with histological hepatic iron overload).

\begin{tabular}{llll}
\hline & \multicolumn{2}{l}{ Histological liver iron score } & Total \\
\cline { 2 - 3 } & 1 & $2-3$ & \\
\hline $\begin{array}{l}\text { Iron within hepatocytes only } \\
\begin{array}{l}\text { Iron within both hepatocytes } \\
\text { and Kupffer cells }\end{array}\end{array}$ & 8 & 1 & 9 \\
$\quad$\begin{tabular}{l} 
Total \\
\hline
\end{tabular} & 11 & 9 & 12 \\
\hline
\end{tabular}

Stainable iron within the Kupffer cells was more frequent in patients with higher hepatic iron scores (Fischer's exact test, $p=0.01 ; R_{S}=0.63, p=0.002$ ).
The mean ferritin $(\mathrm{ng} / \mathrm{ml})$ value was 507 , median 259 , and IQR 641-167. There were no significant differences of ferritin values among different groups of diffuse liver diseases.

\section{Liver R2* measurements}

The mean liver R2* values were $55 \mathrm{~s}^{-1}$ (median 45, IQR $72-37$, range from 26 to $126 \mathrm{~s}^{-1}$ ). These measurements were positively correlated with serum ferritin levels $\left(R_{S}=0.53, p<0.01\right.$; Table 2$)$. This correlation was stronger in cases with ferritin values below $1000 \mathrm{ng} / \mathrm{ml}\left(R_{S}=0.69\right.$, $p<0.01)$.

Liver R2* values were significantly different across the different histological iron grades (Kruskal-Wallis, $p<0.01$ ), increasing with the histological iron grade $\left(R_{S}=0.58\right.$, $p<0.01$; Tables 2 and 3, Fig 2). Using Bonferroni post hoc correction, the $\mathrm{R} 2{ }^{*}$ measurements were significantly different $(p<0.01)$ between grade 0 and $2-3$ and between grade 1 and $2-3$.

A significant correlation was found between liver and spleen R2* measurements $\left(\mathrm{R}_{S}=0.71, p<0.01\right)$ or liver and vertebral bone marrow $\mathrm{R}^{*}{ }^{*}\left(\mathrm{R}_{S}=0.66, p<0.01\right.$; Fig $\left.3 \mathrm{a}-\mathrm{b}\right)$ but not between hepatic and pancreatic $R 2^{*}$ values $\left(R_{S}=0.22\right.$, $p=0.096$; Table 2). Splenic R2* measurements were also correlated with bone marrow $\mathrm{R}^{*}\left(\mathrm{R}_{S}=0.72, p<0.01\right.$; Fig $3 c$, Table 2). Pancreatic R2* values were not correlated with spleen $(p=0.052)$ neither with vertebral bone marrow $(p=0.12) \mathrm{R} 2 *$ measurements nor with serum ferritin levels $(p=0.07)$.

Regarding the iron cellular distribution, the highest R2* values in liver, spleen, and bone marrow were found in patients with stainable iron inside the Kupffer cells (Fig. 4a-c). Patients with iron in Kupffer cells had significantly higher hepatic R2* values than patients with iron only in hepatocytes or no iron overload $(p<0.01)$. There were no significant differences between hepatic $\mathrm{R} 2 *$ measurements in patients with iron only in hepatocytes and patients with no iron overload ( $p>0.05$; Fig 4a). Furthermore, no significant differences were observed in splenic and bone marrow R2* measurements between patients with hepatic iron only in hepatocytes and patients with no hepatic iron overload ( $p>0.05$; Fig 4b,c).

Unfortunately, multifactorial analyses considering the effect of histological iron grade and the iron cellular distribution in the $\mathrm{R} 2 *$ measurements from different tissues could not be performed due to the small number of patients.

\section{Discussion}

Although hepatic iron overload is seen in some cases of chronic diffuse liver diseases, splenic and bone marrow involvement has not been appreciated in the imaging literature; however, the present results, in a cohort of patients with different diffuse liver diseases, show a positive correlation between liver, spleen, and bone marrow iron accumulation, as measured by MRI-derived R2* quantification. Iron liver deposits were histopathologically found either within the hepatocyte compartment only or within 
Table 2

Correlation coefficients between $\mathrm{R} 2 *$ from different tissues, and the serum ferritin levels or hepatic histological iron score.

\begin{tabular}{|c|c|c|c|c|c|c|}
\hline Spearman Correlations & $\begin{array}{l}\text { Histological liver } \\
\text { iron score }(n=56)\end{array}$ & $\begin{array}{l}\text { Ferritin } \\
(n=37)\end{array}$ & $\begin{array}{l}\text { Liver R2* } \\
(n=56)\end{array}$ & $\begin{array}{l}\text { Spleen } \mathrm{R} 2^{*} \\
(n=56)\end{array}$ & $\begin{array}{l}\text { Vertebral bone } \\
\text { marrow R2* }(n=56)\end{array}$ & $\begin{array}{l}\text { Pancreas R2* } \\
(n=56)\end{array}$ \\
\hline Liver R2* & $0.58^{\mathrm{a}}$ & $0.53^{\mathrm{a}}$ & & & & \\
\hline Spleen R2* & $0.52^{\mathrm{a}}$ & $0.49^{\mathrm{a}}$ & $0.71^{\mathrm{a}}$ & & & \\
\hline Vertebral bone marrow $\mathrm{R} 2 *$ & $0.41^{\mathrm{a}}$ & $0.43^{\mathrm{a}}$ & $0.66^{\mathrm{a}}$ & $0.72^{\mathrm{a}}$ & & \\
\hline Pancreas R2* & 0.19 n.s. & 0.30 n.s. & 0.22 n.s. & 0.26 n.s. & 0.21 n.s. & \\
\hline
\end{tabular}

n.s., Not significant.

${ }^{\mathrm{a}} \mathrm{p}<0.01$.

Table 3

R2* from different tissues in the whole study population or stratified by histologic grading of hepatic iron deposition.

\begin{tabular}{|c|c|c|c|c|}
\hline & Liver R2* & Spleen R2* & Vertebra bone marrow R2* & Pancreas R2* \\
\hline In the whole population & $55,45,72-37$ & $40,29,51-18$ & $187,184,217-143$ & $34,32,38-29$ \\
\hline Grade 0 & $42,38,46-34$ & $26,21,29-15$ & $164,157,195-133$ & $32,32,34-29$ \\
\hline Grade 1 & $53,44,64-39$ & $40,35,44-24$ & $192,192,218-150$ & $36,31,39-28$ \\
\hline Grade $2-3$ & $86,85,109-73$ & $69,58,103-38$ & $228,232,260-181$ & $37,35,44-28$ \\
\hline
\end{tabular}

Data are mean, median and IQR $\left(\mathrm{s}^{-1}\right)$.

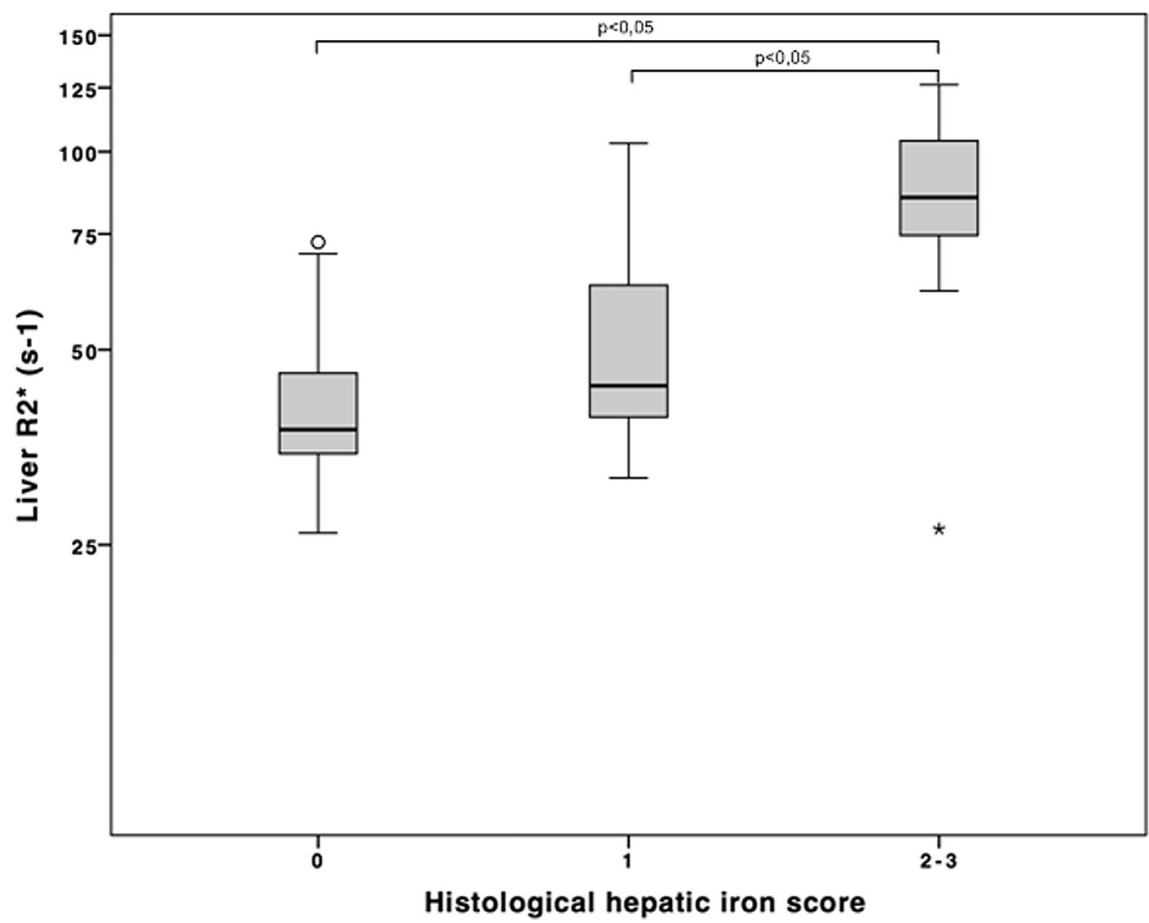

Figure 2 Box and whisker plots of hepatic R2* values measured in all patients in comparison to the histological iron score. The differences of R2* values were significant $(p<0.05)$ between all histological groups of hepatic iron loading. Horizontal lines indicate significant differences between groups, evaluated by post hoc analysis, with Bonferroni adjusted $p$-value for distinguishing different histological grades $<0.05$.

both the hepatocyte and the MPS compartments. The hepatic, splenic, and bone marrow R2* measurements were higher in patients with iron within Kupfer cells, compared to patients with no iron overload. Hepatic R2* measurements were also significantly higher in patients with iron within Kupffer cells than in patients with iron only in hepatocytes. Furthermore, although the difference was not statistically significant, patients with iron within Kupffer cells also had higher splenic and bone marrow R2* measurements than patients with iron only in hepatocytes.
Hepatic R2* measurements were moderately correlated with serum ferritin levels, and this correlation was stronger in patients with serum ferritin under $1,000 \mathrm{ng} / \mathrm{ml}$; however, serum ferritin is limited by its low specificity, despite being widely used as a serological marker of iron burden. This acute phase reactant is elevated in both acute and chronic inflammatory conditions. The liver is the major source of circulating ferritin and minor liver insults might also increase serum ferritin. ${ }^{8}$ Therefore, it is not surprising that the correlation between hepatic $\mathrm{R}^{*}$ measurement and serum 


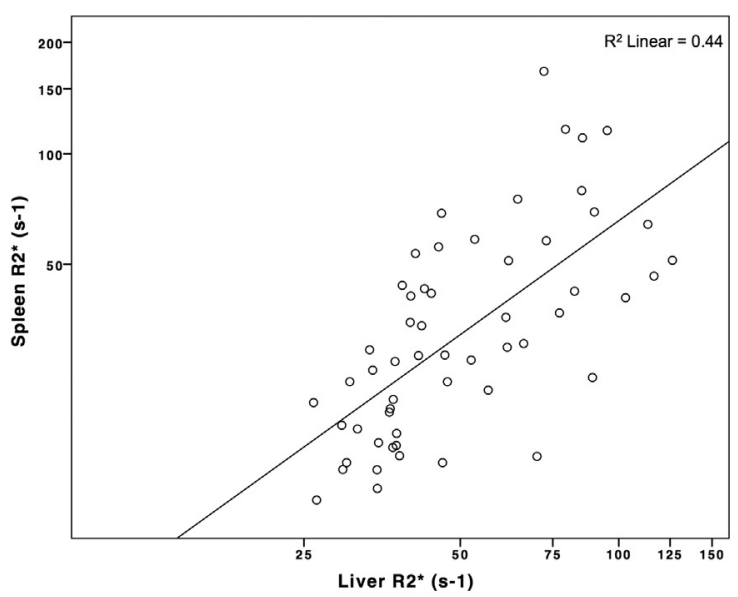

(a)

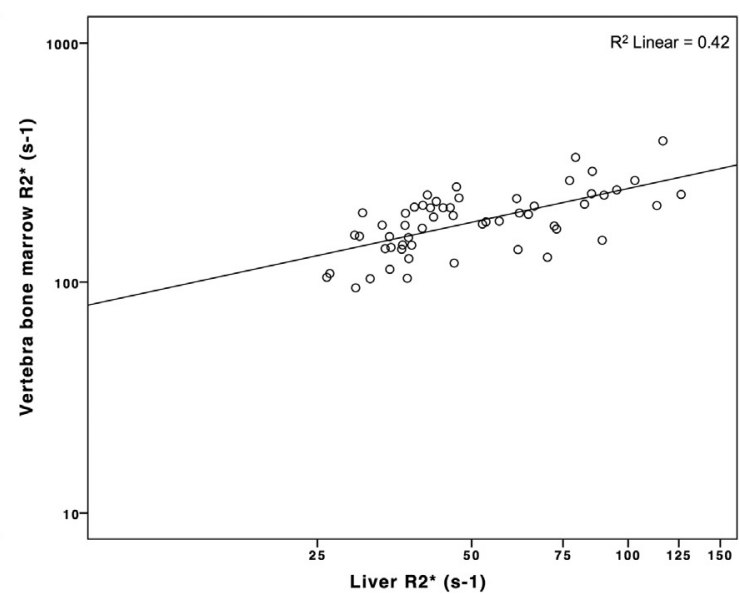

(b)

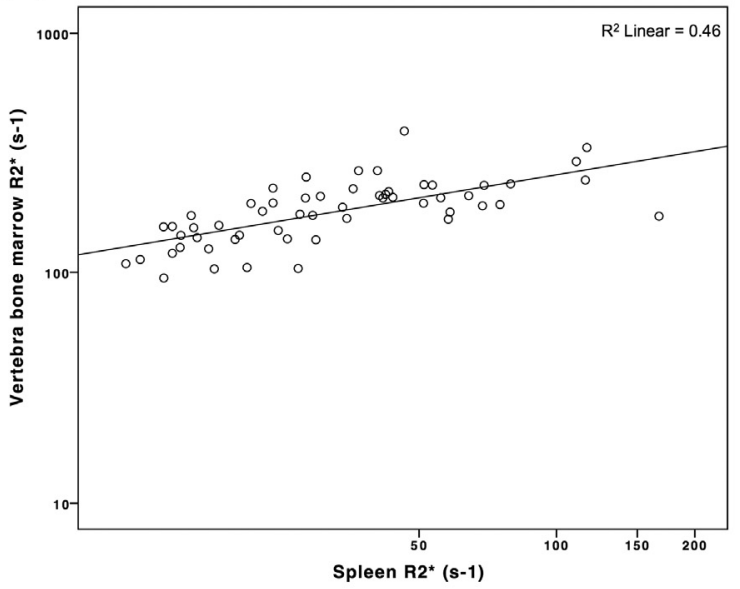

(c)

Figure 3 Scatterplots demonstrating the relationship between hepatic, splenic, and bone marrow R2* measurements. A significant correlation was found between (a) hepatic R2* measurements and spleen $R 2^{*}\left(R_{S}=0.71, p<0.001\right)$, (b) hepatic and vertebral bone marrow $R 2^{*}\left(R_{S}=0.66\right.$, $p<0.001)$, and (c) between spleen and bone marrow $R 2^{*}\left(R_{S}=0.72, p<0.001\right)$. Pancreatic $R 2^{*}$ values were not correlated with any of other tissues.

ferritin levels is affected by other factors besides hepatic iron overload, such as chronic liver inflammatory conditions.

Liver R2* quantification is being increasingly employed in clinical practice as a surrogate biomarker of iron concentration, particularly in patients with hereditary haemochromatosis and haemosiderosis secondary to iron-loading anaemias or chronic transfusion therapies. ${ }^{8,13}$ Abdominal MRI sequences acquired for liver iron concentration quantification can also be used to define and quantify iron accumulation in other tissues, such as pancreas, spleen, and bone marrow. ${ }^{9,10,13-17}$ These MRI images show a different iron load distribution amongst these abdominal organs. The particular organ deposits have been related to the mechanisms of iron flow and tissue overload in the different diseases. In transfusion-related iron overload, excess iron accumulates in the MPS cells within the liver, spleen, and bone marrow, and when these saturate, iron flows to the parenchymal cells of the liver and pancreas. Conversely, in hereditary haemochromatosis, the non-regulated intestinal absorption of iron results in iron accumulation in parenchymal cells of liver and afterwards the pancreas, usually sparing the spleen and bone marrow. ${ }^{2,6,18}$

Mild to moderate iron overload is a common histological finding in chronic liver diseases. The excesses of iron potentially lead to oxidative stress, ${ }^{19}$ which contributes to progression and worsening prognosis of the underlying liver disease $\mathrm{e}^{5}$; however, the mechanisms behind this iron accumulation are still debated. Local pathogenic factors may inhibit the hepatic production of hepcidin, ${ }^{20}$ the key regulator of the intestinal iron absorption and the iron recycling by macrophages. ${ }^{21}$ This inhibition results in systemic iron overload. Conversely, local necro-inflammatory changes might contribute to iron accumulation in the MPS cells. Engulfment of iron-loaded dead bodies by Kupffer cells increases the expression of profibrogenic factors, and persistent activation of these cells promotes the development of fibrosis. ${ }^{22}$ Whether other major MPS-containing organs, such as the spleen or the bone marrow, might also accumulate iron as a systemic response to liver inflammation, is not known and constitutes the focus of the present study. Non-invasive MRI was used to simultaneously 


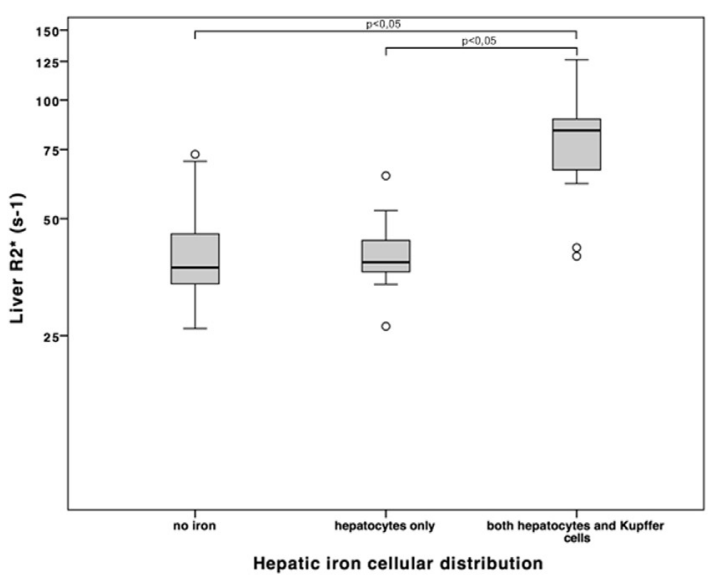

(a)

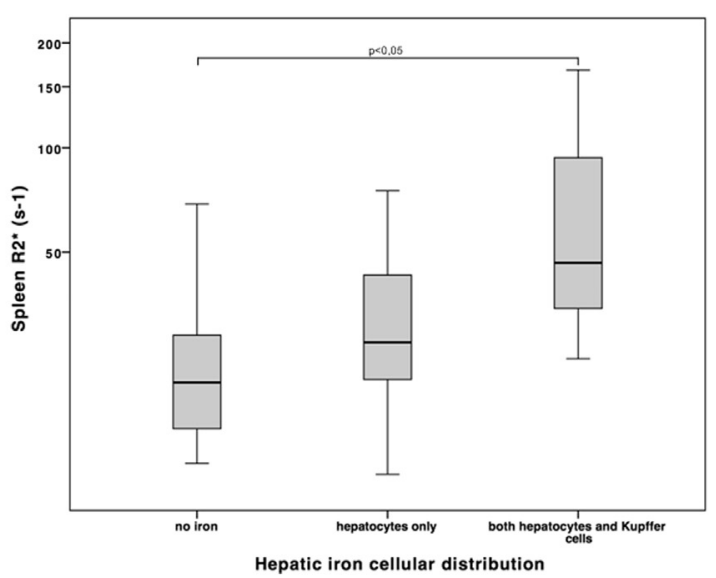

(b)

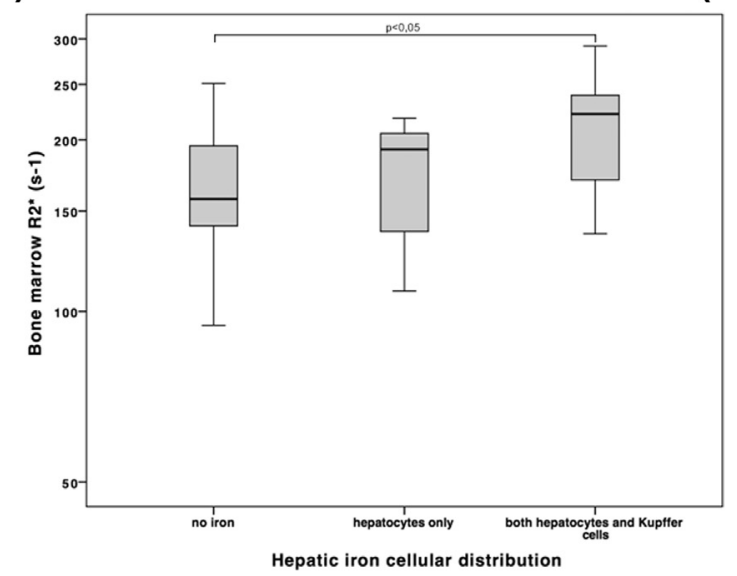

(c)

Figure 4 Box and whisker plots of (a) liver, (b) spleen, and (c) bone marrow R2* values measured in all patients, compared with hepatic histological iron cellular distribution. The highest R2* values in (a) liver, (b) spleen, and (c) bone marrow were found in patients with stainable iron inside Kupffer cells. Horizontal lines indicate significant differences between groups, evaluated by post hoc analysis, with Bonferroni adjusted $p$ value $<0.05$.

quantify R2* values as a biomarker for iron loading in the liver, spleen, bone marrow, and pancreas, in a cohort of patients with chronic diffuse liver diseases. Although previous reports have evaluated the role of R2* MRI quantification in diffuse liver disease, ${ }^{23-26}$ demonstrating a high correlation with liver biopsy iron scoring, the assessment of iron accumulation in organs others than the liver has not been performed in patients with primary chronic liver diseases. In the present study, a positive correlation between liver, splenic, and bone marrow iron accumulation was observed in patients with different diffuse liver diseases. The liver, spleen, and bone marrow R2* values were also shown to be higher in patients who had iron inside Kupffer cells when compared to patients with iron accumulation in hepatocytes. In the present series, there was no relationship between pancreatic and iron deposits in the other organs. This is in agreement with reports of patients with thalassemia and other haematological iron-overload diseases, where such a relationship was not found ${ }^{9}$ or was very poor. ${ }^{10}$ Although there is a report with a significant correlation between the pancreas and liver iron concentrations in children with sickle cell anaemia, this relationship was only established in patients with the highest liver iron concentrations. ${ }^{17}$ In contrast to these patients with ironoverload haematological diseases, patients with primary chronic liver diseases have mild to moderate hepatic iron concentrations. This might explain the lack of association between liver and pancreatic $\mathrm{R}^{*}$ measurements in the present cohort.

The pattern of liver, spleen, and bone marrow $\mathrm{R} 2 *$ relationships in the present cohort of patients with chronic liver diseases is similar to that found previously in transfusion-dependent children with sickle cell anaemia ${ }^{17}$ or thalassemia. ${ }^{9}$ This observation suggests that iron tissue distribution in chronic diffuse liver disorders conveys the general pattern of preferential MPS accumulation, in contrast with the preferential hepatocellular iron accumulation that is typically observed in hereditary haemochromatosis. There were no significant differences in splenic and bone marrow $2^{*}$ measurements in patients with iron in hepatocytes as compared with patients with no iron overload. Conversely, patients with hepatic iron within the Kupffer cells had the highest hepatic, splenic, and bone marrow R2* measurements. 
It should be stressed that a clear distinction between the different iron distribution patterns is an important aspect in the differential diagnosis of iron-overload disorders. The present results suggest that systemic MPS iron accumulation can be observed not only in patients with transfusionrelated iron overload, but also in patients with chronic diffuse liver diseases. The amount of iron deposits in chronic diffuse liver diseases, however, is far below the iron burden observed in transfusion-related iron overload. ${ }^{5}$

In this context, the use of $\mathrm{R} 2 *$ measurements appears as a very promising quantitative novel non-invasive clinical tool. In addition, quantitative iron distribution evaluation by objective R2* MRI-derived methods might be also helpful in clinical trials using iron-removal strategies, such as iron chelators, as well as to investigate the physiopathology of iron metabolism in chronic liver diseases.

The study had some limitations. First, histopathological proof of iron accumulation in the pancreas, spleen, and bone marrow, was not obtained as biopsies in these organs are precluded for ethical constraints. $\mathrm{R} 2 *$ radiological measurements and histological liver biopsy samples evaluation were performed by single observers, and inter- or intra-observer variability values were not assessed; however, in a group of healthy volunteers, $R 2^{*}$ measurements in liver, spleen, and bone marrow showed a strong agreement between different observers and between two different MRI examinations (ICC >0.95) whereas the agreement for pancreatic R2* measurements was good (ICC $>0.78$; unpublished data). Furthermore, the repeatability and accuracy of the $\mathrm{R}^{*}$ method has also been demonstrated in previous reports. $^{27,28}$ The small number of patients in the hepatic iron overload and iron cellular distribution groups is also recognised as a limitation, which has also impaired the use of multifactorial analysis to assess the relative impact of iron overload and cellular distribution of hepatic iron overload. Histological evaluation of iron overload was based on categorical score, which might underestimate the correlation with $\mathrm{R} 2 *$ quantitative continuous values. Unfortunately, quantitative analytical methods for liver iron concentration require a different liver sample and are usually not performed in clinical practice for assessment of diffuse liver diseases. As MRI-estimated liver iron concentrations were not provided, scanner calibration with different iron concentrations was not performed. As the aim was to compare R2* measurements from different tissues, the magnitude of any error or effect would equally affect all the measurements. In addition, no organ was used as an internal reference. In contrast to signal intensity ratio methods, relaxometry methods generate numerical data expressed in international units, and normalisation to a reference organ is not mandatory. Fat can be a confounding factor for R2* measurements due to signal modifications, as fat can be found within the liver, pancreas, and bone marrow. To minimise this bias, a multi-echo chemical-shiftencoded sequence approach was used, considering only the water contribution to the $\mathrm{R} 2 *$ measurements, previously validated in patients with diffuse liver diseases, including non-alcoholic fatty liver disease and non-alcoholic steato- hepatitis. ${ }^{26}$ ROIs measurements were taken from the same hepatic lobe as liver biopsy, in order to minimise the sampling bias. Nevertheless, as iron deposits can be heterogeneously distributed throughout the liver parenchyma and also considering the small size of liver biopsy specimens, it cannot be excluded that the present results might still be affected by some sampling bias.

In summary, a significant correlation between the liver, spleen, and bone marrow iron loading exists in diffuse liver diseases, as assessed by R2* MRI-derived measurements. Pancreatic R2* values were not correlated with iron within the other abdominal organs. Patients with the highest hepatic iron scores and with iron histologically demonstrated within liver Kupffer cells had the highest splenic and bone marrow R2* values, suggesting systemic MPS iron accumulation. Finally, reporting the $\mathrm{R} 2 *$ measurements in other abdominal organs besides the liver bring new insights into the pathophysiology of iron deposition in diverse chronic diffuse liver diseases.

\section{Acknowledgements}

The present study was supported by the Teaching and Research Department of Centro Hospitalar do Porto with a research grant number DEFI:309/12(213-DEFI/251-CES). The funder had no role in study design, data collection and analysis, decision to publish, or preparation of the manuscript. L.M.B. and A.A.B. are co-founders of QUIBIM SME.

\section{References}

1. Nelson JE, Wilson L, Brunt EM, et al. Relationship between the pattern of hepatic iron deposition and histological severity in nonalcoholic fatty liver disease. Hepatology 2010;53:448-57.

2. Sirlin CB, Reeder SB. Magnetic resonance imaging quantification of liver iron. Magn Reson Imaging Clin North Am 2010;18:359-81.

3. Pietrangelo A. Mechanisms of iron hepatotoxicity. J Hepatol 2016:1-2.

4. Turlin B, Mendler MH, Moirand R, et al. Histologic features of the liver in insulin resistance-associated iron overload. A study of 139 patients. Am J Clin Pathol 2001;116:263-70.

5. Pietrangelo Antonello. Iron in NASH, chronic liver diseases and HCC: how much iron is too much? J Hepatol 2009;50:249-51.

6. Queiroz-Andrade M, Blasbalg R, Ortega CD, et al. MRI imaging findings of iron overload. RadioGraphics 2009;29:1575-89.

7. Marti-Bonmati L, Blasco FL. Diffuse parenchymal disease. In: Gourtsoyiannis NC, editor. Clinical MRI of the abdomen. Berlin: Springer; 2011. p. 43-71.

8. Wood JC. Use of magnetic resonance imaging to monitor iron overload. Hematol Oncol Clin N Am 2014;28:747-64.

9. Papakonstantinou O, Alexopoulou E, Economopoulos N, et al. Assessment of iron distribution between liver, spleen, pancreas, bone marrow, and myocardium by means of R2 relaxometry with MRI in patients with $\beta$-thalassemia major. J Magn Reson Imaging 2009;29:853-9.

10. Pfeifer CD, Schoennagel BP, Grosse R, et al. Pancreatic iron and fat assessment by MRI-R2* in patients with iron overload diseases. J Magn Reson Imaging 2014;42:196-203.

11. Deugnier Y, Turlin B. Pathology of hepatic iron overload. WJG 2007;13:4755-60.

12. Martí-Bonmatí L, Alberich-Bayarri A, Sánchez-González J. Overload hepatitides: quanti-qualitative analysis. Abdom Imaging 2011;37:180-7.

13. Wood JC. Guidelines for quantifying iron overload. Hematol Am Soc Hematol Educ Program 2014;2014:210-5. 
14. Papakonstantinou $\mathrm{O}$, Ladis $\mathrm{V}$, Kostaridou $\mathrm{S}$, et al. The pancreas in $\beta$ thalassemia major: MRI imaging features and correlation with iron stores and glucose disturbances. Eur Radiol 2006;17:1535-43.

15. Papakonstantinou O, Drakonaki EE, Maris T, et al. MRI imaging of spleen in beta-thalassemia major. Abdom Imaging 2015;40:2777-82.

16. Noetzli LJ, Papudesi J, Coates TD, et al. Pancreatic iron loading predicts cardiac iron loading in thalassemia major. Blood 2009;114:4021-6.

17. Wood JC, Cohen AR, Pressel SL, et al. Organ iron accumulation in chronically transfused children with sickle cell anaemia: baseline results from the TWiTCH trial. Br J Haematol 2015;172:122-30.

18. Piperno A. Classification and diagnosis of iron overload. Haematologica 1998;83:447-55.

19. Pietrangelo A. Genetics, genetic testing, and management of haemochromatosis: 15 years since hepcidin. Gastroenterology 2015;149. 1240-1251.e1244.

20. Girelli D, Nemeth E, Swinkels DW. Hepcidin in the diagnosis of iron disorders. Blood 2016;127:2809-13.

21. Ganz T. Hepcidin and iron regulation, 10 years later. Blood 2011;117:4425-33.

22. Guicciardi ME. Apoptosis: a mechanism of acute and chronic liver injury. Gut 2005;54:1024-33.
23. Kühn J-P, Hernando D, del Rio AM, et al. Effect of multipeak spectral modeling of fat for liver iron and fat quantification: correlation of biopsy with MRI imaging results. Radiology 2012;265:133-42.

24. Chandarana H, Lim RP, Jensen JH, et al. Hepatic iron deposition in patients with liver disease: preliminary experience with breath-hold multiecho T2*-weighted sequence. AJR Am J Roentgenol 2009;193:1261-7.

25. Banerjee R, Pavlides M, Tunnicliffe EM, et al. Multiparametric magnetic resonance for the non-invasive diagnosis of liver disease. $J$ Hepatol 2014;60:69-77.

26. França M, Alberich-Bayarri A, Martí-Bonmatí L, et al. Accurate simultaneous quantification of liver steatosis and iron overload in diffuse liver diseases with MRI. Abdom Radiol 2017;42:1434-43.

27. Restaino G, Meloni A, Positano V, et al. Regional and global pancreatic T*2 MRI for iron overload assessment in a large cohort of healthy subjects: normal values and correlation with age and gender. Magn Reson Med 2010;65:764-9.

28. Wood JC, Zhang P, Rienhoff $\mathrm{H}$, et al. Liver MRI is more precise than liver biopsy for assessing total body iron balance: a comparison of MRI relaxometry with simulated liver biopsy results. Magn Reson Imaging 2015;33:761-7. 